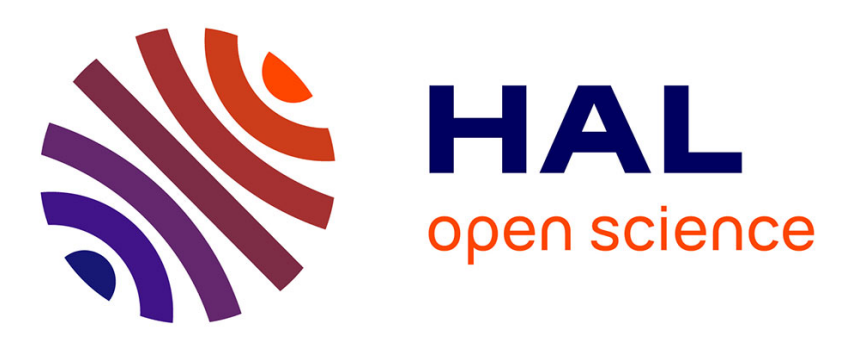

\title{
A new mathematical model for car drivers with spatial preview
}

Wenjuan Jiang, Carlos Canudas de Wit, Olivier Sename, Jonathan Dumon

\section{To cite this version:}

Wenjuan Jiang, Carlos Canudas de Wit, Olivier Sename, Jonathan Dumon. A new mathematical model for car drivers with spatial preview. IFAC WC 2011 - 18th IFAC World Congress, Aug 2011, Milan, Italy. pp.10206. hal-00581800

\section{HAL Id: hal-00581800 https://hal.science/hal-00581800}

Submitted on 31 Mar 2011

HAL is a multi-disciplinary open access archive for the deposit and dissemination of scientific research documents, whether they are published or not. The documents may come from teaching and research institutions in France or abroad, or from public or private research centers.
L'archive ouverte pluridisciplinaire HAL, est destinée au dépôt et à la diffusion de documents scientifiques de niveau recherche, publiés ou non, émanant des établissements d'enseignement et de recherche français ou étrangers, des laboratoires publics ou privés. 


\title{
A new mathematical model for car drivers with spatial preview
}

\author{
Wenjuan Jiang, Carlos Canudas-de-Wit, Olivier Sename and Jonathan Dumon
}

\begin{abstract}
A mathematical driver model is given in the spacial equation form, which takes into account the previewed information of the path. Optimal control method is considered for the ideal no time-delay case and Lyapunov-Krasovskii functional approach is applied to deal with drivers' reaction time-delay. For both cases, exponential stability is guaranteed for the closedloop system.

Keywords: driver model, time-delayed system, optimal control, Lyapunov-Krasovskii functional, LMI
\end{abstract}

\section{INTRODUCTION}

Many researches have been working on driver behaviors and various models have been proposed to avoid traffic accidents and facilite car driving. Furthermore, efficient and feasible driver model is also highly demanded by the intelligent transportation system as the Intelligent Vehicle Highway Systems of USA, the Program for European Traffic with Highest Efficiency and Unprecedented Safety.

In the psychologic point of view, driver behaviors can be described as a set of dynamic models like Kalman filters sequenced together by a Markov chain ( Liu A. and Pentland A. [1997], Kuge N. et al. [2000]). As the human behavior is more complex, these research results are difficult for real application but they bring out some knowledge to be embedded of man's mental functions.

The most studied driver model is to treat the driver-vehicle as an open or closed-loop control system. Driver model is required to represent the pilot experience of drivers, however, as human is a highly self-learning system which can adapted to the variable circumstances, most driver models are concerned with steering control.

In this research direction, two basic categories of driver models have been proposed: compensation tracking models and preview tracking models. The former takes into account the error between the actual driving state information and the expected one. PID compensation model proposed by Iguchi M. [1959] and Crossover model by Ashkens I.L. and McRuer D.t. [1962] are the first type of models. These models can be used to estimate the stability of the closed-loop control system with perturbation but it cannot be used for the fast-driving case.

The preview tracking models use the lateral position of the vehicle at some specified distance or distances ahead of the vehicle as the feedback term ( Modjtahedzadeh A. and Hess R. [1993], Guo K. and Guan H. [1993]). As the future path input has been introduced into the system,

\footnotetext{
0 W.-J. Jiang was with Control System department, GIPSALab, Grenoble, France. W.-J. Jiang is now with Nantong University, China. C. Canudas-de-Wit, O. Sename and J. Dumon are with Control System department, GIPSA-Lab, Grenoble, France. Email: \{wenjuan.jiang, carlos.canudas-de-wit, olivier.sename and jonathan.dumon\}@gipsa-lab.grenoble-inp.fr
}

better tracking accuracy can be obtained. The research of the preview driver model is generally developed with four different methods: transfer function, optimal control, fuzzy control and neural network methods. Early researches use transfer function to present the preview, forward correction and feedback estimation so as to imitate the driver preview, performance and perception. The parameters of the transfer function cannot be directly calculated, which limits its application.

The optimal control concept was first presented by Sheridan T.B. [1966]. The driver/vehicle tracking problem is considered as a local optimal preview model. The driver always estimates the forthcoming road condition and tries to minimize the vehicle tracking error. In the exiting literature, previewed effect is considered in the time horizon, i.e., the preview time is considered (see MacAdam C.C. [1980]). For these models, as the speed of vehicles varies, so the previewed distance is uncertain.

Driver fuzzy control model describes human operating performance and represents to some degree human thoughts (Kageyama I. and Pacejka H.B. [1992]). And finally, there are neural network approaches to driver modeling for vehicle control (Thorpe C. et al. [1992], Wells G. et al. [1996]) where driver model steering angle output is mapped from vision based road views. These two last methods need a very large number of experimental data.

In this paper, we propose a new preview optimal driver model, in which the future path information is directly concerned in the space-horizon model. The new mathematical driver model will be considered in the closedloop control system composed of the vehicle and the path information. The information of the available previewed path is taken into account in the closed-loop control design. The space state equation is analyzed to simplify the model. The remainder of the paper is organized as follows: The vehicle kinematic model is considered in Section II. In Section III, first, a driver mathematical model which takes into account the information of the driver previewed information is presented, then optimal control method is considered for the ideal no time-delay case, followed by simulation results. Section IV is devoted to experimented driver model. Lyapunov-Krasovskii functional approach is applied to deal with drivers' reaction time-delay. In Section $\mathrm{V}$, some comparison of the two cases is made. 


\section{VEHICLE MODEL}

\subsection{Global coordinate model}

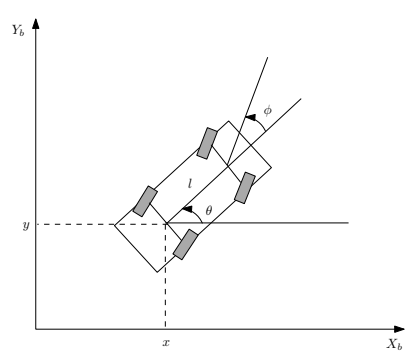

Fig. 1. The global coordinate system for the car

Fig. (1) shows the exact position and orientation of the car in some global coordinate system which can be described by four main variables. The $(x, y)$ coordinates give the location of the center of the rear axle. The car angle with respect to the $\mathrm{x}$-axis is given by $\theta$. The steering wheel angle with respect to the car longitudinal axis is given by $\phi$.

The complete kinematic model is given as Mellodge $\mathrm{P}$. [2002]:

$$
\left[\begin{array}{c}
\dot{x} \\
\dot{y} \\
\dot{\theta} \\
\dot{\phi}
\end{array}\right]=\left[\begin{array}{l}
\cos (\theta) \\
\sin (\theta) \\
\frac{\tan (\phi)}{l} \\
0
\end{array}\right] v_{1}+\left[\begin{array}{l}
0 \\
0 \\
0 \\
1
\end{array}\right] v_{2}
$$

where $v_{1}$ is the linear velocity of the rear wheels and $v_{2}$ is the angular velocity of the steering wheels.

\subsection{Path coordinate model}

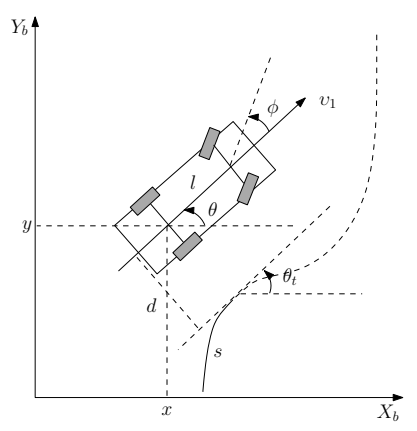

Fig. 2. The path coordinates for the car

The sensors cannot detect the car location with respect to some global coordinates but the desired path. Therefore, a more useful model is one that describes the car behavior in terms of the path coordinates.

The path coordinates are shown in Fig. (2). The perpendicular distance between the rear axle and the path is given by $d$. The angle between the car and the tangent to the path is $\tilde{\theta}=\theta-\theta_{t}$. The distance traveled along the path starting at some arbitrary initial position is given by $s$, the arc lengh. The car kinematic model in terms of the path coordinates is given ( Canudas de wit C. et al. [1996]):

$$
\begin{aligned}
\dot{s} & =\frac{v_{1} \cos (\tilde{\theta})}{1-d c(s)} \\
\dot{d} & =v_{1} \sin (\tilde{\theta}) \\
\dot{\tilde{\theta}} & =v_{1}\left(\frac{\tan (\phi)}{l}-\frac{c(s) \cos (\tilde{\theta})}{1-d c(s)}\right)
\end{aligned}
$$

where $c(s)$ is the path curvature and is defined as

$$
c(s)=\frac{d \theta_{t}}{d s}
$$

Note that the parameter $v_{2}$ is excluded from the model equation, which will facilitate the control problem.

\subsection{Model transformations}

Suppose that the driver well tracks the path, that mean, in the neighborhood of $(d=0, \tilde{\theta}=0)$, tangent linearization of the last two equations in (2) gives:

$$
\begin{aligned}
\dot{d} & =v_{1} \tilde{\theta} \\
\dot{\tilde{\theta}} & =v_{1}\left(\frac{\phi}{l}-\frac{c(s)}{1-d c(s)}\right)
\end{aligned}
$$

To simplify the model, rewrite the temporal equation (4) into the spacial form with $\frac{\partial s}{\partial t}=\left|v_{1}\right|$ and the assumption $v_{1}>0$, then

$$
\begin{aligned}
& d^{\prime}=\frac{\partial d}{\partial s}=\tilde{\theta} \\
& \tilde{\theta}^{\prime}=\frac{\partial \theta}{\partial s}=\frac{\phi}{l}-\frac{c(s)}{1-d c(s)}
\end{aligned}
$$

Introducing the auxiliary control variable

$$
\nu=\frac{\phi}{l}-f(s), f(s)=\frac{c(s)}{1-d c(s)},
$$

the equation (5) becomes

$$
\begin{aligned}
& d^{\prime}=\tilde{\theta} \\
& \tilde{\theta}^{\prime}=\nu
\end{aligned}
$$

Define the system state $z$ as the following equation

$$
z=\left[\begin{array}{l}
d \\
\tilde{\theta}
\end{array}\right]
$$

then the system model can be written as

$$
z^{\prime}=A z+B \nu
$$

where

$$
z^{\prime}=\frac{\partial z}{\partial s}, A=\left[\begin{array}{ll}
0 & 1 \\
0 & 0
\end{array}\right], B=\left[\begin{array}{l}
0 \\
1
\end{array}\right]
$$

\section{DRIVER MODEL}

The driver is considered as a feedback controller which takes into account of the path previewed information. Fig. 3 presents the structure of the closed-loop driver tracking system. $\tau_{1}$ and $\tau_{2}$ represent respectively the driver's brain response delay and physical action time, then the timedelay in the spatial model can be expressed as $\delta_{1}=v_{1} *$ $\tau_{1}$. In the following part, first the ideal case without consideration of time-delay in the closed-loop system will be concerned by applying optimal preview control method; then, the time-delay from the driver's reaction will be added to the system, in which case both the stability and the performance of the system will be considered. 


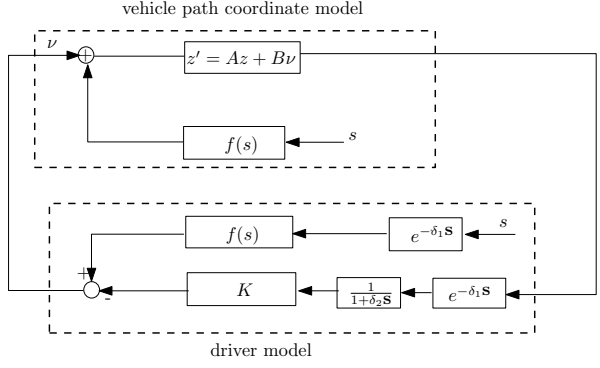

Fig. 3. The closed-loop system of driver tracking model

\subsection{Optimal preview control}

Consider the following condition (Anderson B.D.O. and Moore J.B. [1969]) to be minimized:

$$
J=\int_{0}^{\infty}\left(z^{T} Q z+r \nu^{2}\right) e^{2 \lambda s} d s
$$

where the accessible previewed distance $L=1 / \lambda>0$ and

$$
Q=\left[\begin{array}{ll}
\left|v_{1}\right| & 0 \\
0 & \left|v_{1}\right|
\end{array}\right]
$$

Assuming for the moment that a linear constant control law will minimize $J$, all the poles of the closed-loop system must lie in the half plane $R e<-\lambda$, i.e., closedloop pole constraint exits. The complete controllability of equation (9) assures that there is some control for which $J$ is finite. Consequently the minimum value of $J$ is finite. The assumption that the control law is linear and constant implies that $z$ and $\nu$ behave exponentially to ensure finiteness of $J, z$ and $\nu$ must decay faster than $e^{-\lambda s}$, which, in turn, implies the closed-loop pole constraint. To minimize equation (11) subject to the conditions of equation (9), set

$$
\begin{aligned}
& x=z e^{\lambda s}, \\
& u=\nu e^{\lambda s}
\end{aligned}
$$

then equation (9) is equivalent to

$$
x^{\prime}=(A+\lambda I) x+B u
$$

Then minimization with respect to equation (13) of equation (11) is equivalent to minimization with respect to equation (14)

$$
J=\int_{0}^{\infty}\left(x^{T} Q x+r u^{2}\right) d s
$$

in the following senses:

(a) The minimum value of equation (11) (expressed in terms of $z_{0}$ ) is the same as the minimum value of equation (15) (expressed in terms of $x_{0}$, taking account of $x_{0}=$ $\left.e^{\lambda s} z_{0}\right)$.

(b) If $u=g(x)$ is the optimal control for equation (14) and (15), $\nu=e^{-\lambda s} g\left(z e^{\lambda s}\right)$ is the optimal control for equation (9) and (11), and conversely.

For equations (14) and (15), the optimal control is

$$
u=-K x
$$

where $K=\frac{1}{r} B^{T} P$ and $P$ is the unique nonnegative definite solution of the Riccati equation

$$
P(A+\lambda I)+\left(A^{T}+\lambda I\right) P-\frac{1}{r} P B B^{T} P+Q=0
$$

(Equivalently, $P$ could be found as the limiting solution of a Riccati equation.) The second remark above then yields that the optimal control for equation (9) and (11) is

$$
\nu=-K z
$$

Thus, the construction of the desired control law is essentially no more difficult than for the case when $\lambda=0$.

With the equation (6) and (18), the control $\phi$ applied to the vehicle can be rewritten as follows:

$$
\phi=l(f(s)-K z) \text {. }
$$

\subsection{Tracking simulation results}

First the ideal case is considered, i.e., driver's reaction delay is not presented. For comparison, $\lambda=0.025$ and $\lambda=2$ are taken respectively as the long previewed distance $40 \mathrm{~m}$ and the shorter one $0.5 \mathrm{~m}$, the vehicle speed $v_{1}=$ $3 \mathrm{~m} / \mathrm{s}$. The simulation results are showed in the Fig. 4,5 and 6 . As for the two cases, the vehicle well tracks the path, there is not much difference. The average control $\phi=0.0263$ radian and the average steering input deviation $\dot{\phi}=0.024$.

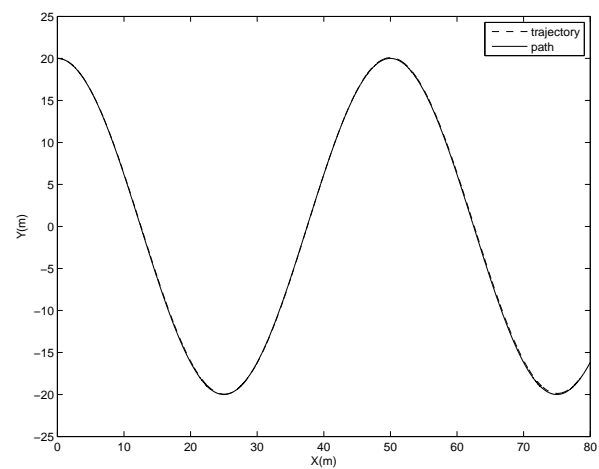

Fig. 4. The path tracking with $\lambda=0.025$
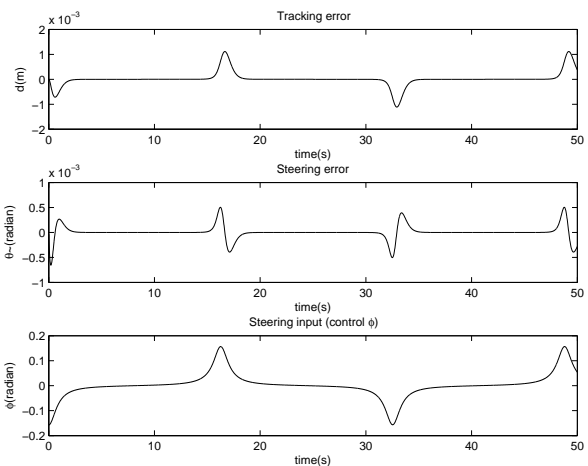

Fig. 5. The state variables $(\mathrm{d}, \tilde{\theta})$ and the control $(\phi)$ with $\lambda=0.025$ (Optimal)

As there is always driver brain response delay $\tau_{1}$ and driver action time $\tau_{2}$ showed in the Fig. 3, for the same simulation condition as path curves and vehicle speed, a constant time-delays $\left(\tau_{1}=0.1 \mathrm{~s}\right.$ and $\left.\tau_{2}=0.1 \mathrm{~s}\right)$ are added to the control, then the following simulation results in Fig. 7 and 8 are obtained. For the case with a longer previewed distance, there are oscillations but the system is stable while the system becomes unstable with short previewed 

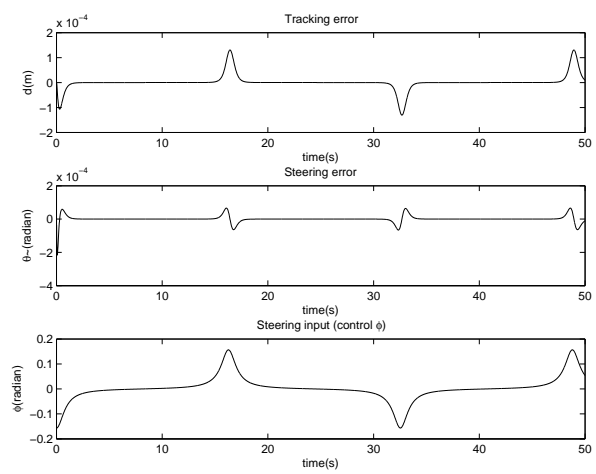

Fig. 6. The state variables $(\mathrm{d}, \tilde{\theta})$ and the control $(\phi)$ with $\lambda=2$ (Optimal)

distance. That means, due to driver's reaction delay, there is a previewed distance limit for tracking the path.
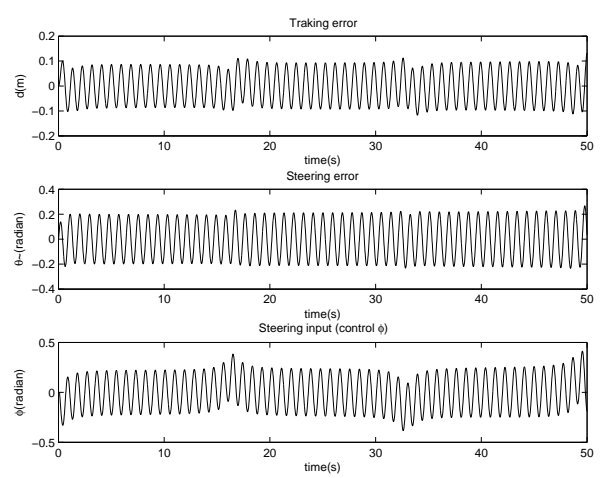

Fig. 7. $(\mathrm{d}, \tilde{\theta})$ and $(\phi)$ with $\lambda=0.025, \tau_{1}=0.1 s$ (Optimal)
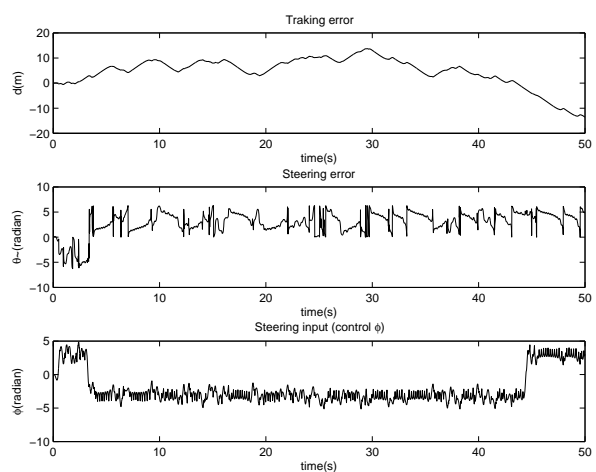

Fig. 8. $(\mathrm{d}, \tilde{\theta})$ and $(\phi)$ with $\lambda=2, \tau_{1}=0.1 s$ (Optimal)

\section{EXPERIMENTED DRIVER MODEL}

As an experimented driver always takes into account his reaction delay, it is necessary to take this time-delay into consideration in the driver model. So the system equation 9 with time-delay $\delta$ is written as

$$
z^{\prime}=A z+B v(s-\delta) .
$$

\subsection{Lyapunov-Krasovskii functional approach}

Rewrite equation (20) with time-delayed control $v=$ $-K_{d} z(s-\delta)$ and the same parameter change techniques as showed in the precedent section:

$$
x^{\prime}=(A+\lambda I) x-e^{\lambda \delta} B K_{d} x(s-\delta)
$$

If the asymptotic stability of the system (21) can be guaranteed, exponential stability for the orignal timedelayed system can be obtained. The following result ensures the stability of the system in the presence of timedelay.

Proposition: If for some positive scalars $\lambda$ and $\varepsilon$, there exist positive symmetric matrices $0<P_{1}=P_{1}^{T}, P_{2}, R=R^{T}$ and $M$ with propre dimension such that the LMI (23) with the notation given in (24) is feasible, then the gain:

$$
K_{d}=M P_{2}^{-1}
$$

exponentially stabilizes the system $z^{\prime}=A z-B K_{d} z(s-\delta)$ with the decay rate $\lambda$.

$$
\begin{aligned}
& \Phi= \\
& {\left[\begin{array}{ccc}
\Phi_{11} & (*) & (*) \\
\Phi_{21} & \Phi_{22} & (*) \\
-\delta e^{\lambda \delta} M^{T} B^{T} & -\varepsilon \delta e^{\lambda \delta} M^{T} B^{T} & -\delta R
\end{array}\right]<0,}
\end{aligned}
$$

$\Phi_{11}=P_{2}^{T}(A+\lambda I)^{T}+(A+\lambda I) P_{2}-e^{\lambda \delta} M^{T} B^{T}-e^{\lambda \delta} B M$, $\Phi_{21}=P_{1}-P_{2}^{T}+\varepsilon(A+\lambda I) P_{2}-\varepsilon e^{\lambda \delta} B M$,

$\Phi_{22}=-\varepsilon P_{2}-\varepsilon P_{2}^{T}+\delta R$.

In order to prove the stability of the closed-loop system, the following lemma on the stability conditions for constant time-delay systems is first stated.

Lemma 1. (Fridman E. and Shaked U. [2001]) The system

$$
x^{\prime}=A_{0} x+A_{1} x(s-h),(h>0)
$$

is asymptotically stable if there exist $0<P_{1}=P_{1}^{T}, P_{2}, P_{3}$ and $R=R^{T}$ with propre dimension that satisfy the following LMI:

$$
\Phi=\left[\begin{array}{ccc}
\left(\sum_{i=0}^{1} A_{i}^{T}\right) P_{2}+P_{2}^{T}\left(\sum_{i=0}^{1} A_{i}\right) & (*) & (*) \\
P_{1}-P_{2}+P_{3}^{T}\left(\sum_{i=0}^{1} A_{i}\right) & -P_{3}-P_{3}^{T}+h R & (*) \\
h A_{1}^{T} P_{2} & h_{1} A_{1}^{T} P_{3} & -h R
\end{array}\right]<0,
$$

proof (sketching proof of Lemma 1) Represent the timedelayed system in the equivalent descriptor form:

$$
x^{\prime}=y, 0=-y+\left(A_{0}+A_{1}\right) x-A_{1} \int_{s-h}^{s} y(\eta) d \eta .
$$

Considering the extension of the classical, quadratic Lyapunov-Krasovskii functionals (LKF):

$$
V\left(s, x_{s}, \dot{x}_{s}\right)=\left[\begin{array}{ll}
x^{T} & y^{T}
\end{array}\right] E P\left[\begin{array}{l}
x \\
y
\end{array}\right]+\int_{-\delta}^{0} \int_{s+\xi}^{s} y^{T}(\eta) R y(\eta) d \eta d \xi
$$

where

Note that

$$
E=\left[\begin{array}{ll}
I & 0 \\
0 & 0
\end{array}\right], P=\left[\begin{array}{ll}
P_{1} & 0 \\
P_{2} & P_{3}
\end{array}\right]
$$

$$
\begin{aligned}
& \frac{d}{d s}\left[\begin{array}{ll}
x^{T} & y^{T}
\end{array}\right] E P\left[\begin{array}{l}
x \\
y
\end{array}\right]=2 x_{T} P_{1} x^{\prime}=2\left[\begin{array}{ll}
x^{T} & y^{T}
\end{array}\right] P^{T}\left[\begin{array}{l}
x^{\prime} \\
0
\end{array}\right] \\
& =2\left[\begin{array}{ll}
x^{T} & y^{T}
\end{array}\right] P^{T}\left[\begin{array}{l}
y \\
-y+\left(A_{0}+A_{1}\right) x-A_{1} \int_{s-h}^{s} y(\eta) d \eta
\end{array}\right]
\end{aligned}
$$

Differentiating (28) in $s$ and applying (30) we obtain 


$$
\frac{d V(s)}{d s} \leq\left[\begin{array}{ll}
x^{T} & y^{T}
\end{array}\right] \Phi\left[\begin{array}{l}
x \\
y
\end{array}\right]<0
$$

if the LMI (26) is feasible. This concludes the proof.

To applying Lemma 1, we rewrite (21) in the equivalent descriptor form:

$x^{\prime}=y, 0=-y+\left(A+\lambda I-e^{\lambda \delta} B K_{d}\right) x-e^{\lambda \delta} B K_{d} \int_{s-\delta}^{s} y(\eta) d \eta$

and use some change of variables $P_{3}=\varepsilon P_{2}, \varepsilon>0$, the LMI

(23) can be obtained (Jiang W.-J. [2009]).

\subsection{Tracking simulation results}

In Table 1, we can see that the shorter the distance previewed, the higher gain will be needed. It is logical to the case of real driving. When the driver could not see very far because of the fog or the sharp couverture of the road, he has to control the wheel in high frequency. To compare optimal control and LKF methods, the gain for $\tau_{1}=0$ are calculated by the two methods. LKF approach with LMI conditions is mainly used for dealing with the stability of time-delayed systems but may have some conservatism.

From Table 1, we can also see that the time-delay has a great effect on the stability of the system. when the driver is inexperienced or with slow reactions, he needs more previewed distance to make decision. For example, the minimum $L$ for the case $\tau_{1}=0.1$ is $0.78 m$, for $\tau_{1}=0.2$ is $1.67 \mathrm{~m}$ and for $\tau_{1}=0.3$ at least $2.7 \mathrm{~m}$.

First, the simulations with a same time-delay $\left(\tau_{1}=0.1 s\right)$ but different previewed distances $(\lambda=0.025$ and $\lambda=$ $1)$ are carried out. The simulation results are showed respectively in Fig. 9 and 11. From the Table 2, the average tracking errors $|d|$ for the two case is almost the same but the average derivation of the control $\phi$ for the case with a far preview is much smaller.
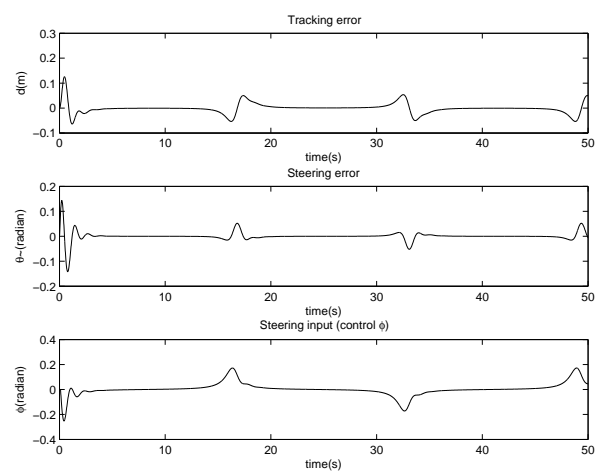

Fig. 9. $(\mathrm{d}, \tilde{\theta})$ and $\phi$ with $\lambda=0.025, \tau_{1}=0.1 s(\mathrm{LKF})$

Now, consider the driver with longer delays $\left(\tau_{1}=0.3 \mathrm{~s}\right)$ for two previewed case $(\lambda=0.025$ and $\lambda=0.37)$. Similar results are showed in Fig. 11 and 12.

\section{COMPARISON BETWEEN LQR AND LKF}

The direct Lyapunov method is a powerful tool for studying systems stability, it is useful for determination of
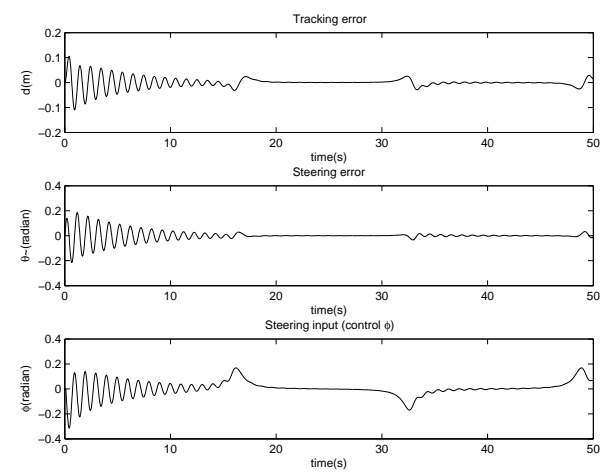

Fig. 10. $(\mathrm{d}, \tilde{\theta})$ and $\phi$ with $\lambda=1, \tau_{1}=0.1 s(\mathrm{LKF})$
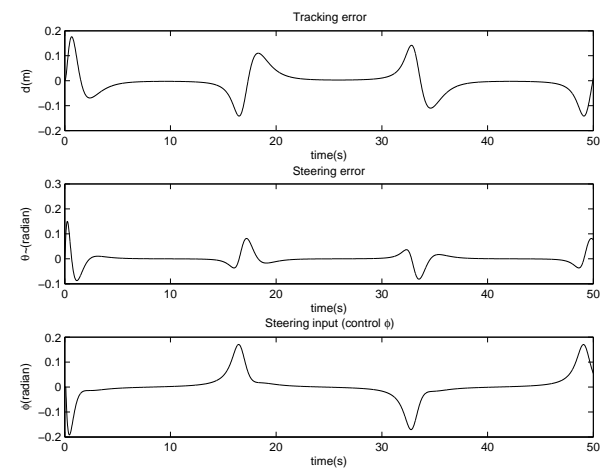

Fig. 11. $(\mathrm{d}, \tilde{\theta})$ and $\phi$ with $\lambda=0.025, \tau_{1}=0.3 s(\mathrm{LKF})$
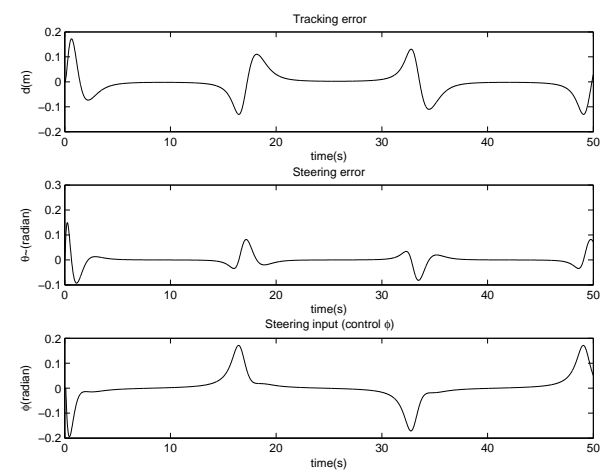

Fig. 12. $(\mathrm{d}, \tilde{\theta})$ and $\phi$ with $\lambda=0.37, \tau_{1}=0.3 s(\mathrm{LKF})$

\begin{tabular}{ccccc}
\hline$\tau_{1}(s)$ & $\lambda$ & average $|d|(m)$ & average $|\phi|($ radian $)$ & average $|\dot{\phi}|$ \\
\hline 0.1 & 0.025 & 0.011 & 0.0263 & 0.033 \\
0.1 & 1 & 0.011 & 0.0378 & 0.12 \\
0.3 & 0.025 & 0.0727 & 0.03 & 0.044 \\
0.3 & 0.37 & 0.069 & 0.032 & 0.051 \\
\hline
\end{tabular}

Table 2. Previewed distance and time-delay effects (LKF)

robust stability conditions. For the familiar linear ordinary differential equation

$$
x^{\prime}=A_{0} x, A_{0} \in R^{n \times n}, x \in R^{n} .
$$

Consider a Lyapunov function $V_{1}$ in a quadratic form

$$
V_{1}=x^{T} P x
$$

Then the necessary and sufficient conditions for asymptotic stability of system (32) can be formulated in terms of existence of a positive definite matrix $P$ for any symmetric $Q>0$ : 


\begin{tabular}{|c|c|c|c|c|c|c|}
\hline \multicolumn{4}{|c|}{ Optimal control } & \multicolumn{3}{|c|}{ LKF method } \\
\hline$\lambda$ & $\mathrm{L}(\mathrm{m})$ & $\mathrm{K}$ & $K_{d}\left(\tau_{1}=0\right)$ & $K_{d}\left(\tau_{1}=0.1\right)$ & $K_{d}\left(\tau_{1}=0.2\right)$ & $K_{d}\left(\tau_{1}=0.3\right)$ \\
\hline 2 & 0.5 & {$\left[\begin{array}{ll}19 & 8.7\end{array}\right]$} & {$\left[\begin{array}{ll}13.3 & 7.3\end{array}\right]$} & - & - & - \\
\hline 1 & 1 & {$\left[\begin{array}{ll}6.85 & 5.2\end{array}\right]$} & {$\left[\begin{array}{ll}4.73 & 4.2\end{array}\right]$} & {$\left[\begin{array}{ll}1.73 & 2.24\end{array}\right]$} & - & - \\
\hline 0.5 & 2 & {$\left[\begin{array}{lll}3.56 & 3.72\end{array}\right]$} & {$\left[\begin{array}{ll}2.36 & 2.82\end{array}\right]$} & {$\left[\begin{array}{ll}1.16 & 1.96\end{array}\right]$} & {$\left[\begin{array}{lll}0.47 & 1.13\end{array}\right]$} & - \\
\hline 0.1 & 10 & {$\left[\begin{array}{ll}2 & 2.75\end{array}\right]$} & [1.29 1.88$]$ & {$\left[\begin{array}{ll}0.75 & 1.63\end{array}\right]$} & {$\left[\begin{array}{ll}0.35 & 1.04\end{array}\right]$} & {$\left[\begin{array}{lll}0.179 & 0.727\end{array}\right]$} \\
\hline 0.025 & 40 & {$\left[\begin{array}{ll}1.8 & 2.59\end{array}\right]$} & {$\left[\begin{array}{ll}1.17 & 1.72\end{array}\right]$} & {$\left[\begin{array}{ll}0.68 & 1.54\end{array}\right]$} & {$\left[\begin{array}{ll}0.30 & 0.96\end{array}\right]$} & {$\left[\begin{array}{lll}0.176 & 0.732\end{array}\right]$} \\
\hline 0.01 & 100 & {$\left[\begin{array}{ll}1.76 & 2.56\end{array}\right]$} & {$\left[\begin{array}{ll}1.14 & 1.69\end{array}\right]$} & {$\left[\begin{array}{ll}0.67 & 1.53\end{array}\right]$} & {$\left[\begin{array}{ll}0.29 & 0.94\end{array}\right]$} & {$\left[\begin{array}{ll}0.17 & 0.69\end{array}\right]$} \\
\hline
\end{tabular}

$$
A_{0}^{T} P+P A_{0}=-Q
$$

But for the equation with delay the situation is different. Consider the system (25) with a constant time-delay, which can be transformed in the following form. Here and below the notation $A=A_{0}+A_{1}$ will be used.

$$
\dot{x}(t)=A x(t)-A_{1} \int_{t-h}^{t} \dot{x}(s) d s
$$

Lemma 2. ( Kolmanovskii V.B. and Richard J.P. [1999]) Assume that for some symmetric matrices $R>0, Q>0$ there exits a solution $P>0$, of the Riccati equation

$$
A^{T} P+P A+h P A_{1} R^{-1} A_{1}^{T} P+h A_{1}^{T} R A_{1}=-Q
$$

then system $(25)$ is asymptotically stable.

proof (sketching proof of Lemma 2) Consider a LKF $V_{2}=x^{T} P x+\int_{-h}^{0} \int_{s+\xi}^{s} x^{T}(\eta) R x(\eta) d \eta d \xi+h \int_{s-h}^{s} x^{T}(\eta) A_{1} R A_{1} x(\eta) d \eta$ to guarantee the asymptotical stability of the system (25), the condition $\dot{V}_{2}<0$ has to be proved.

$$
\begin{gathered}
\dot{V}_{2}=x^{T}\left[A^{T} P+P A+h P A_{1} R^{-1} A_{1}^{T} P+h A_{1}^{T} R A_{1}\right] x \\
-\int_{s-h}^{s}\left[A_{1}^{T} P x(\eta)+R \dot{x}(\eta)\right]^{T} R^{-1}\left[A_{1}^{T} P x(\eta)+R \dot{x}(\eta)\right] d \eta
\end{gathered}
$$

This derivative is negative under the assumption of (34). This concludes the proof.

From the proof, we can see that the stability condition is less conservative for small delay values. Compared to the method LKF with LMI solution, to resolve the Riccati equation (34) for gain calculation is more complicated.

\section{CONCLUSION}

A mathematical driver model in the spatial equation form has been introduced for analysis of drivers' behavior. In the model, a previewed distance is taken into account. First, optimal control is applied. For the ideal case without driver's reaction delay concerned, for both long distance preview and shorter one, the vehicle tracks well the path. whereas, when time-delay added into the system, too short preview distance cause the instability of the system. The simulation result corresponds with the real driving experience. Then, Lyapunov-Krasovskii functional approach is applied deal with stability problem with the driver's delay. In this case, when the delay becomes greater, the longer preview distance will be needed. The average derivation of the steering input $\phi$ is calculated for different preview distance as well as the time-delay cases, which well verifies our driver model. The main contribution of this model is that the preview effect only depends on the path information and it does not affected by the vehicle speed, so the result is more neutral.

\section{REFERENCES}

Canudas de wit C., Siciliano B., and Bastin G. Theory of robot control. Springer-Verlag, 1996.

Guo K. and Guan H. Modelling of driver/vehicle directional control system. Vehicle System Dynamics, (22): 141-184, 1993.

Kolmanovskii V.B. and Richard J.P. Stability of some linear systems with delay. IEEE Transactions on Automatic Control, 44(5):984-989, 1999.

Kuge N., Yamamura T., Shimoyama O., and Liu A. A driver behavior recognition method based on a driver model framework. SAE transactions, 109:469-476, 2000.

Liu A. and Pentland A. Recognizing driver intentions with hidden markov models. Nissan Technical Review, 41:53-56, 1997.

MacAdam C.C. An optimal preview control for linear systems. Journal of Dynamic Systems, Measurement and Control, Transactions of the ASME, 102(3):188190, 1980.

Modjtahedzadeh A. and Hess R. A model of driver steering control behavior for use in assessing vehicle handling qualities. ASME J. Dynam. Syst. Meas. Contr., 15: 456464, Sept. 1993.

Sheridan T.B. Three models of preview control. IEEE Trans. on Human Factors in Electronics, HFE-7(2), 1966.

Wells G., Venaille C., and Torras C. promising research vision-based robot positioning using neural networks. Image vision computing, 14, 1996.

Anderson B.D.O. and Moore J.B. Linear system optimisation with prescribed degree of stability. Proc. IEEE, 116:2083-2087, 1969.

Ashkens I.L. and McRuer D.t. A theory of handling qualities derived from pilot/vehicle system consideration. Aerospace Engineering, (2), 1962.

Fridman E. and Shaked U. New bounded real lemma representations for time-delay systems and their applications. IEEE Trans. on Automatic control, 46(12):1973-1979, December 2001.

Iguchi M. A study of manual control. Journal of mechanic Society of Japan, 62(481), 1959.

Jiang W.-J. A contribution to control and observation of networked control systems. Ph.D thesis, June 2009.

Kageyama I. and Pacejka H.B. On a new driver model with fuzzy control. Vehicle System Dynamics, 20:314324, 1992.

Mellodge P. . Feedback control for a path following robotic car. thesis, April 2002.

Thorpe C. , Hebert M., Kanade T., and Shafer C. The new generation system for the cmu navlab. Vision-Based Vehicle Guidance, September 1992. 